\section{Fully digital implant-prosthetic reconstruction in dental office}

T. Dostalova, S. Halamova, P. Kriz

Department of Stomatology Charles University 2nd Faculty of Medicine and University Hospital in Motol, Prague, Czech Republic
IMPLANT THERAPY OUTCOMES, PROSTHETIC ASPECTS

\section{Results}

\section{Abstract}

Intraoral impression, occlusion field registration, 3D print model and CAD/CAM implant prosthetic rehabilitation started to be an important technique in dental practice. Implant-supported CAD/CAM crowns achieved the best possible long-term results from an aesthetic point of view with the least possible distress for the patient. The goal of our study is to demonstrate connection of intraoral scanning with $3 \mathrm{D}$ print and with CAD/CAM crowns preparation.

\section{Background and Aim}

Precise documentation of the oral cavity and proportions in the face area is crucial not only in dentistry, e.g. prosthetics, orthodontics or orthognathic surgery planning, but also in implantology. Current imaging methods together with dental impressions enable a satisfactory imaging of tissues, which is required for a given purpose. Mainly dental of tissues, which is required for a given purpose. Mainly dental
impressions and plaster casts are used for reproduction of the situation in the oral cavity. Usage of an intraoral scanner is one of the new possibilities how to document and preserve 3D data about patients. Intraoral scanning is a non-invasive method, well tolerated by patients and more time effective compared to traditional dental impressions with dental impression trays and appropriate impression material. A record from the intraoral scanner consists of single pictures and videosequences.

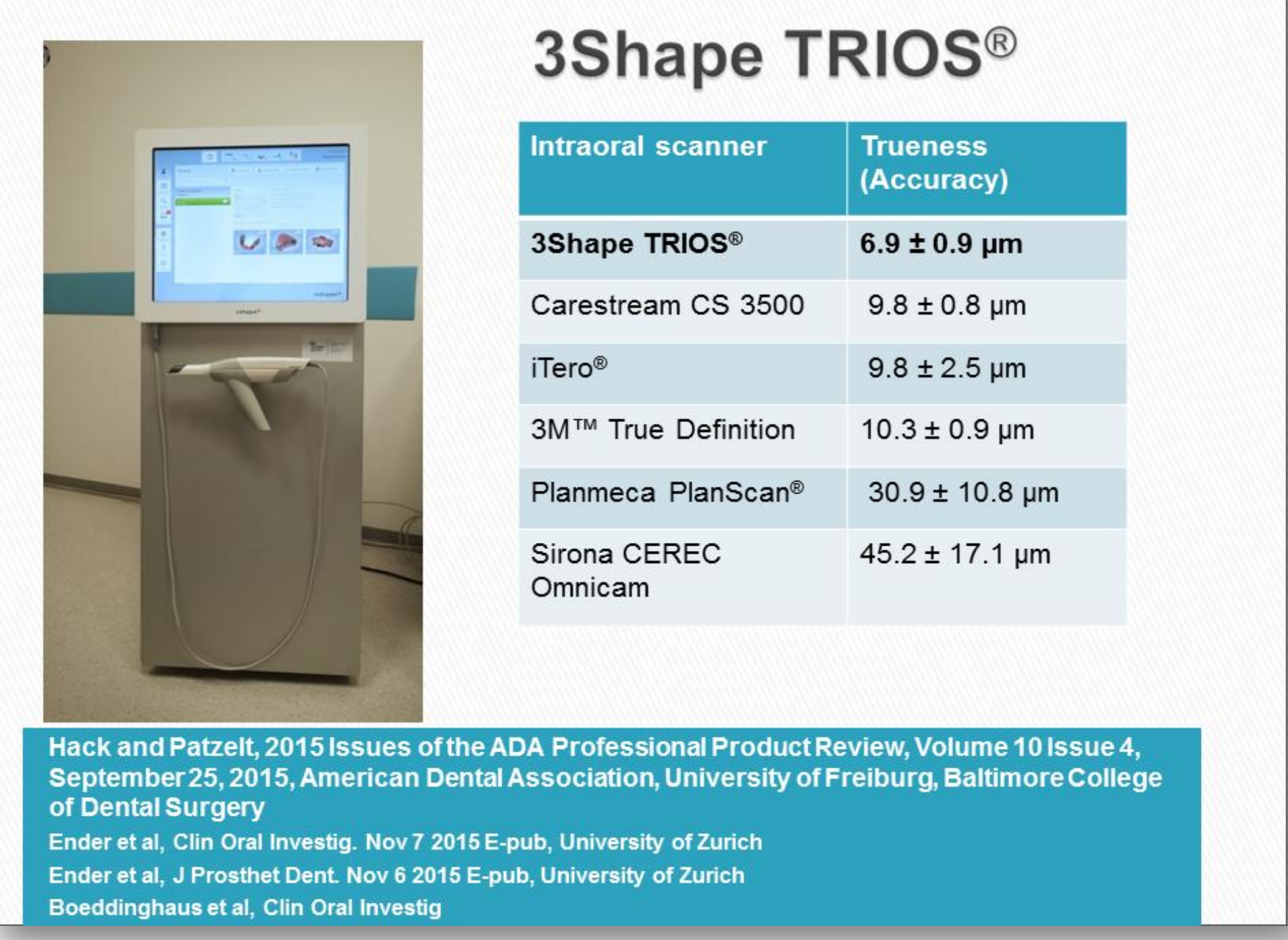

The aim of study was to evaluate the precision of fully digital implantprosthetic reconstruction ASTRA TECH Implant System ${ }^{\text {TM }}$ EV in dental office using Trios $₫ 3$ Shape Dental Systems digital intraoral scanner, 3D print and CAD /CAM techniques to create prosthetics components.

\section{Methods and Materials}

Prospective study of 20 implants AstraTech EV was prepared. The range of patients at implants surgery moves from 18 to 35 years. Multidisciplinary therapy by the surgeon, orthodontist, and prosthodontist before implant insertion was monitored. 3Shape TRIOS $\otimes 3$ - a three in one digital impression solution and 3D printed model were used before computer-aided design and computer-aided manufacturing. The CAD /CAM techniques - abutments (Dentsply Int.), Zircon Zahn (Prettauß Zirconia) were used to establish a framework. The aesthetic appearance was checked mainly from the point of view vertical position of framework and loss of bone marginal support. For every patient we checked step upper and lower jaw digital impression, occlusion, create prosthetics components including digital models in articulator, and CAD/CAM reconstruction. Rescanning of 3D print model and X-ray image after reconstruction helped to evaluate the results.
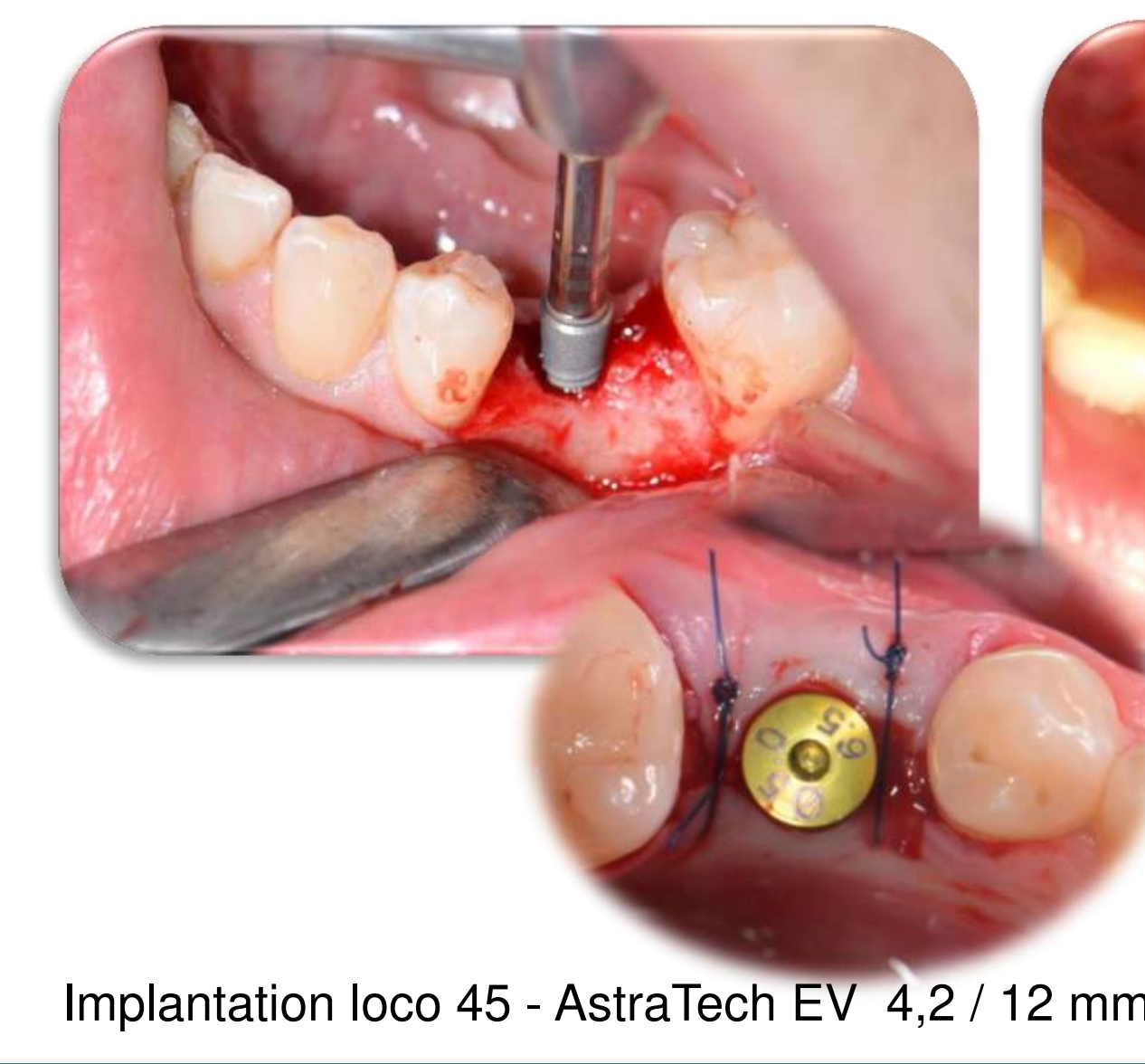

Implantation loco 45 - AstraTech EV 4,2 / 12 mm.
Case reports demonstrate the functional and esthetic results. A good or acceptable aesthetic appearance at the last observation was found in $98 \%$ subjects. 3D reconstruction of occlusion helped us to prepare optimal occlusion without problems with registration material. Also vertical position of the implant-supported crown was precise registered especially in subjects with no incisor contact. Rescanning process and Xray image after reconstruction confirmed the acceptance of digital impression and 3D print model in clinical praxis.

Patient rehabilitation step by step:

31years old women - generally healthy - AstraTech EV 4,2 / 12 mm loco 36
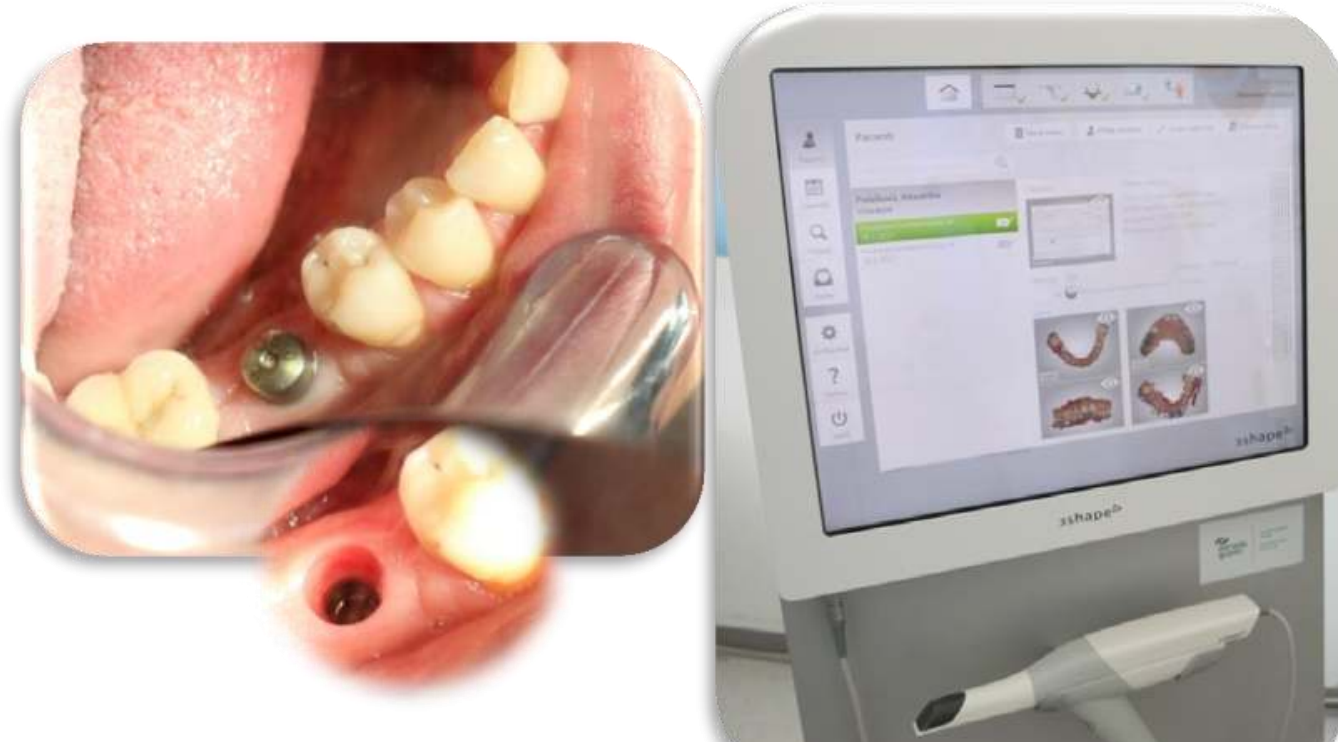

3Shape TRIOS $® 3$ - see scanning process. https://youtu.be/7iMP ZiMeyw
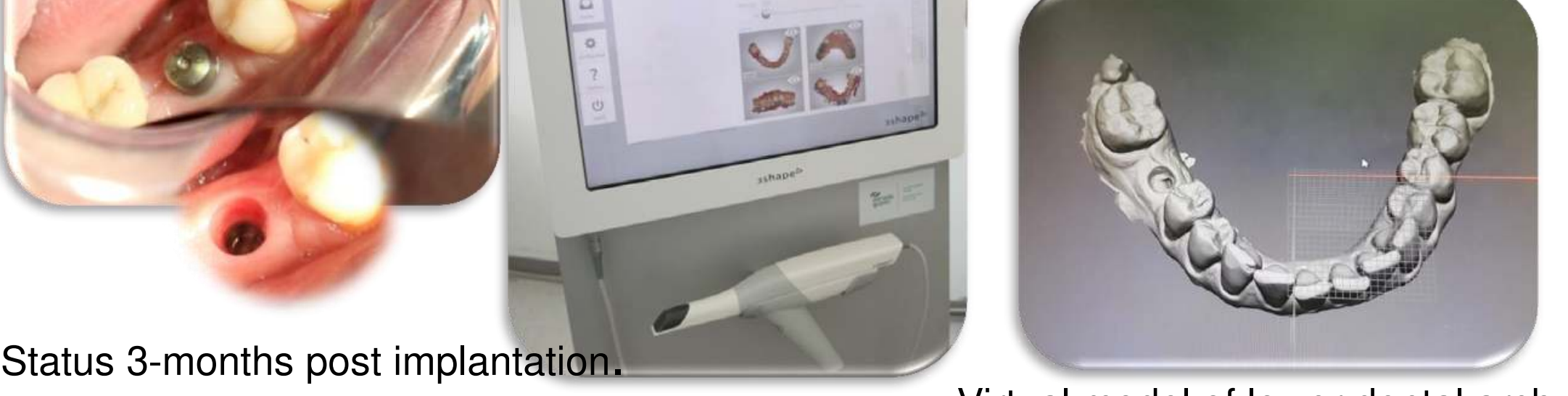

Virtual model of lower dental arch

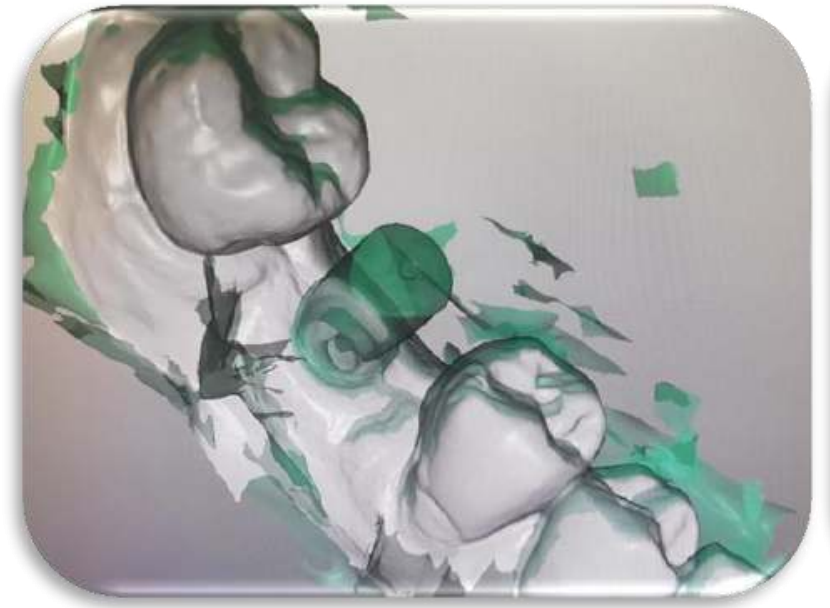

Implant position in 3D virtual model with scan body.

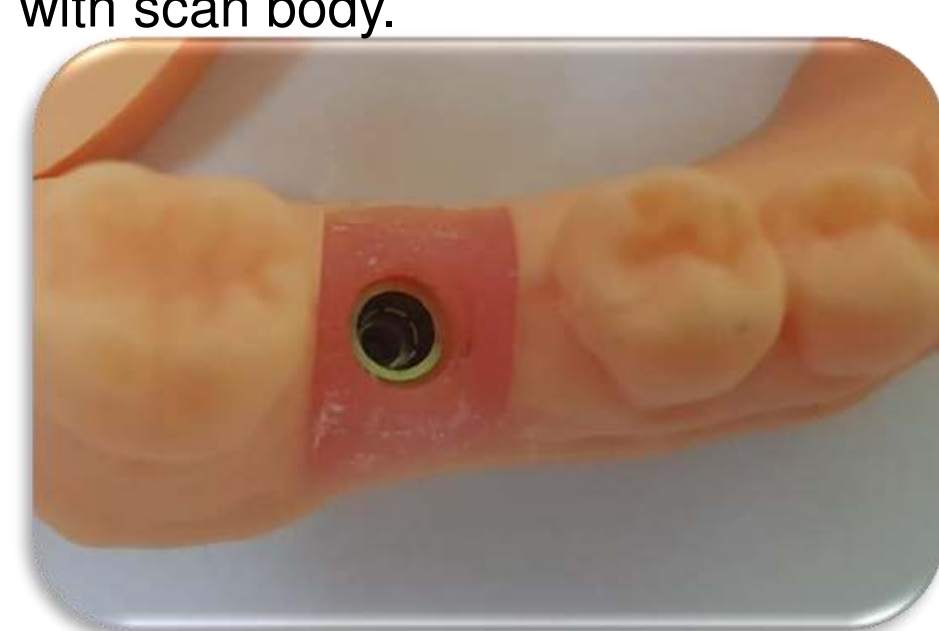

3D print model with gingival mask.

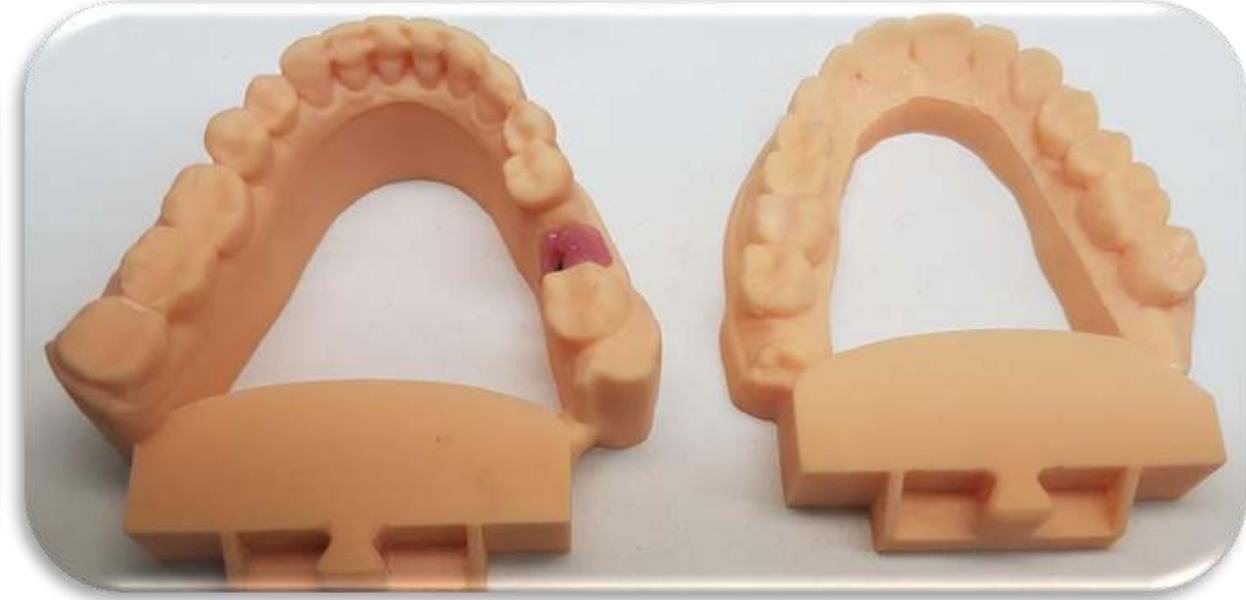

3D print - upper and lower model.

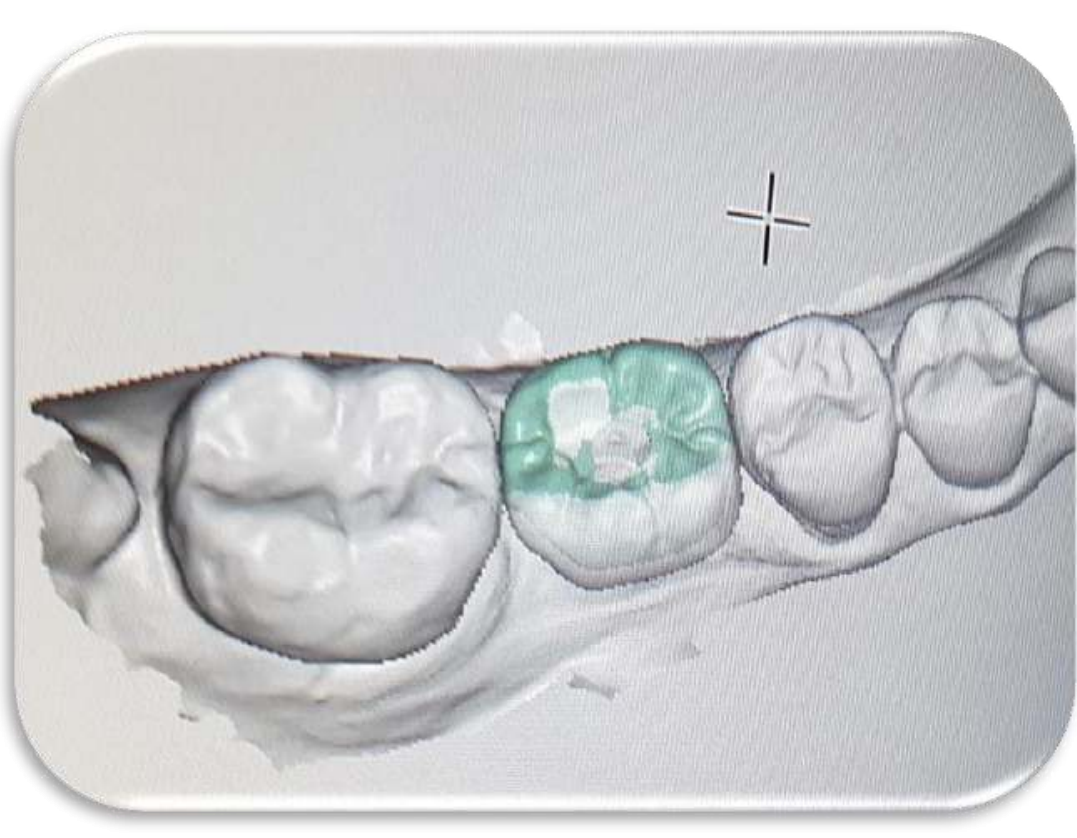

Implant hybrid base in situ.

Computer-Aided Crown Design

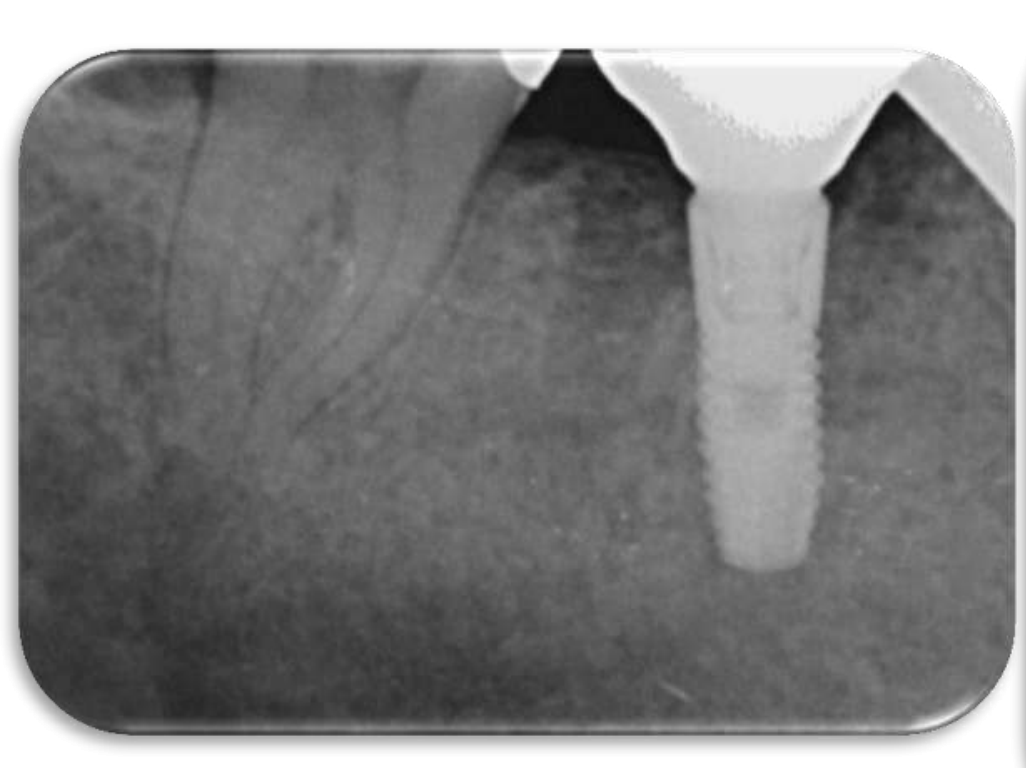

X-ray image after reconstruction.
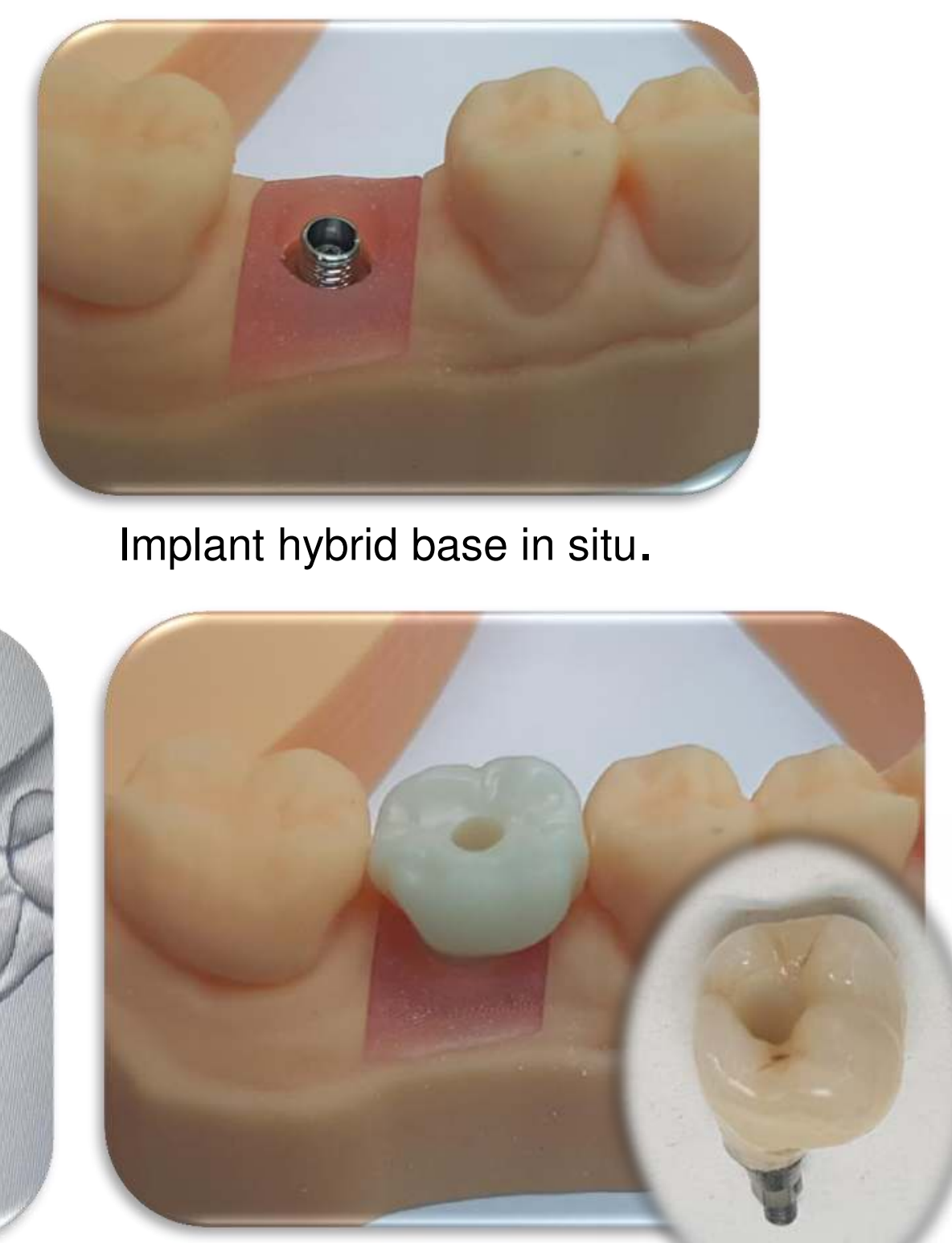
Full ceramic crown with hybrid base.

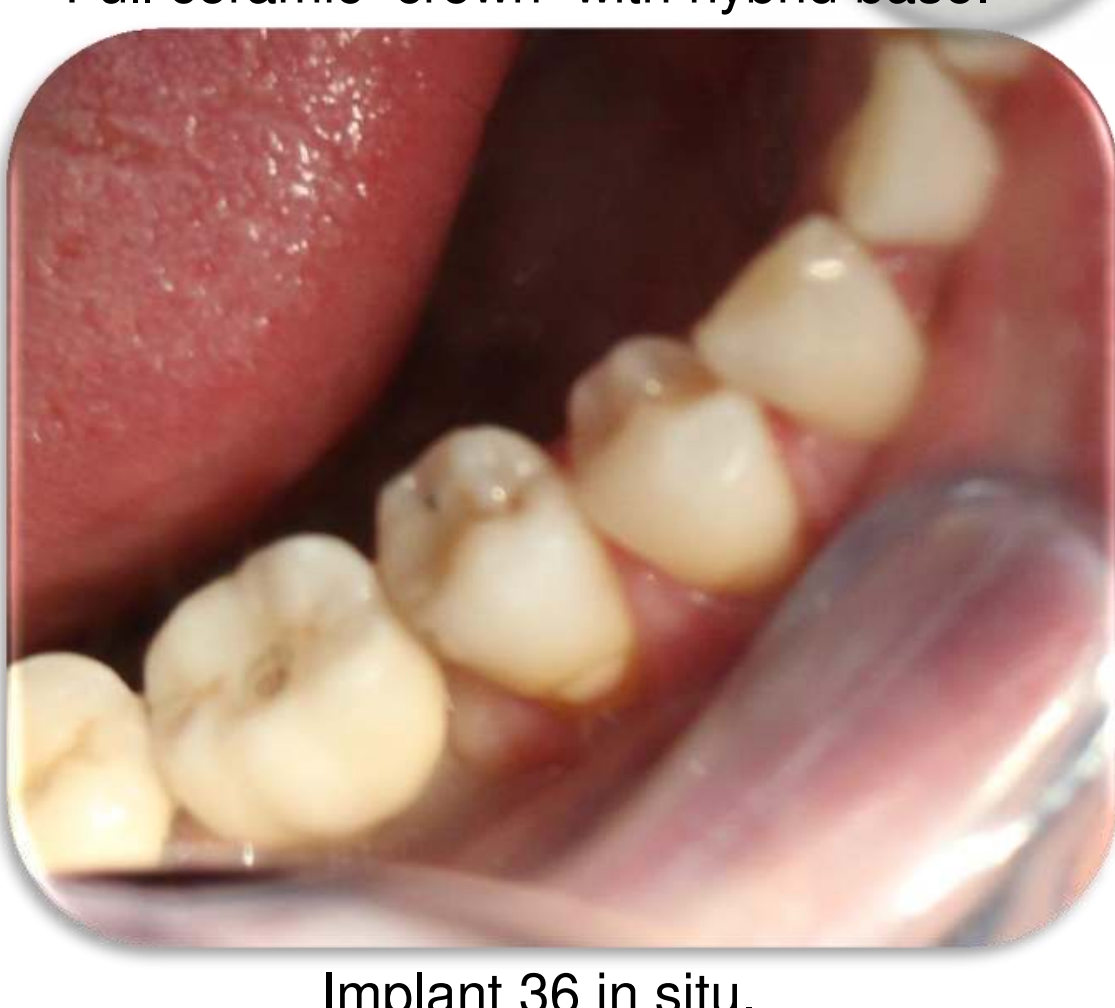

Implant 36 in situ.

\section{Conclusions}

The aesthetic and functional result has been achieved. Very important was that during patient rehabilitation no need to change occlusal field adjustment. No biological and mechanical complications were found. Fully digital implant-prosthetic reconstruction provided a smooth, complication free and time effective treatment alternative to the conventional materials and methods. This system guaranties long-term and high functional and aesthetic stability of results in dental office. 